\title{
A NOTE ON BOUNDARY VALUE PROBLEMS FOR THE HEAT EQUATION IN LIPSCHITZ CYLINDERS
}

\author{
RUSSELL M. BROWN AND ZHONGWEI SHEN
}

(Communicated by J. Marshall Ash)

\begin{abstract}
We study the initial Dirichlet problem and the initial Neumann problem for the heat equation in Lipschitz cylinders, with boundary data in mixed norm spaces $L^{q}\left(0, T, L^{p}(\partial \Omega)\right)$.
\end{abstract}

\section{INTRODUCTION}

Let $\Omega$ be a bounded Lipschitz domain in $\mathbf{R}^{n}, n \geq 3$, and, for $0<T<\infty$, let $\Omega_{T}=\Omega \times(0, T)$ be a Lipschitz cylinder. Consider the heat equation

$$
\frac{\partial u}{\partial t}-\Delta u=0 \quad \text { in } \Omega_{T} \text {. }
$$

The purpose of this note is to study the solvability of the initial Dirichlet problem

$$
\left\{\begin{array}{l}
\left.u\right|_{\Sigma_{T}}=g \in L^{q}\left(0, T, L^{p}(\partial \Omega)\right), \\
\left.u\right|_{t=0}=0
\end{array}\right.
$$

and the initial Neumann problem

$$
\left\{\begin{array}{l}
\left.\frac{\partial u}{\partial N}\right|_{\Sigma_{T}}=g \in L^{q}\left(0, T, L^{p}(\partial \Omega)\right), \\
\left.u\right|_{t=0}=0
\end{array}\right.
$$

where $\Sigma_{T}=\partial \Omega \times(0, T)$ denotes the lateral boundary of $\Omega_{T}$ and $N$ denotes the outward unit normal to $\partial \Omega$. We prove that the initial Dirichlet problem is solvable for $2 \leq p<\infty, 1<q<\infty$ (Theorem 1.1) and that the initial Neumann problem is solvable for $1<p \leq 2,1<q<\infty$ (Theorem 1.2). Moreover, the solutions can be represented by heat potentials and the ranges of $p, q$ are optimal.

In the case of $p=q$, the initial Dirichlet problem was solved in [FS] for $2 \leq p<\infty$ and the initial Neumann problem was solved in [B1, B2] for $1<p \leq 2$.

Our results are established by the method of layer potentials (see [B1, B2, DK, S, V]). For the initial Neumann problem, the existence of solutions is

Received by the editors March 3, 1992.

1991 Mathematics Subject Classification. Primary 35K20.

The first author was partially supported by the NSF and the Commonwealth of Kentucky through the Kentucky EPSCoR program. The second author was partially supported by the NSF. 
reduced to the invertibility of the boundary potential operator $\frac{1}{2} I+K$ on $L^{q}\left(0, T, L^{p}(\partial \Omega)\right)$. In [B1, B2] it is shown that $\frac{1}{2} I+K$ is invertible on $L^{p}\left(0, T, L^{p}(\partial \Omega)\right)$ for $1<p \leq 2$. To establish the invertiblity of $\frac{1}{2} I+K$ on $L^{q}\left(0, T, L^{p}(\partial \Omega)\right)$ we use the vector-valued Calderón-Zygmund machinery. This leads to the study of layer potentials for the Helmholtz-type equation:

$$
-\Delta u+(1+i \tau) u=0 \quad \text { in } \Omega, \tau \in \mathbf{R} .
$$

We are able to show that $\left(\frac{1}{2} I+K\right)^{-1}$ is associated with an $L(\mathbf{B})$-valued Calderón-Zygmund kernel where $L(\mathbf{B})$ denotes the space of bounded linear operators on $\mathbf{B}=L^{p}(\partial \Omega)$. A standard Calderón-Zygmund argument then yields that $\left(\frac{1}{2} I+K\right)^{-1}$ is bounded on $L^{q}\left(0, T, L^{p}(\partial \Omega)\right)$ for $1<p \leq 2,1<q<\infty$. The result for the initial Dirichlet problem follows by duality.

We remark that the methods of this paper provide a simpler proof of Theorem 2.7 and its corollaries in [BS2]. In this earlier paper, we used estimates in mixed $L^{p}$-spaces in the course of studying the initial Dirichlet problem for parabolic systems in (ordinary) $L^{p}$-spaces. It was this application that led us to the research reported here.

Our main results are stated and proved in $\S 1$. Throughout this note, $C$ and $c$ denote constants which depend at most on $n, p, q, T$ and the Lipschitz constant of $\Omega$.

\section{1. $L^{p, q}$-ESTIMATES FOR THE HEAT EQUATION}

Let $L^{p, q}\left(\Sigma_{T}\right)=L^{q}\left(0, T, L^{p}(\partial \Omega)\right)$ denote the space

$$
\left\{f:\|f\|_{L^{p, q}\left(\Sigma_{T}\right)}=\left(\int_{0}^{T}\left(\int_{\partial \Omega}|f(P, t)|^{p} d P\right)^{q / p} d t\right)^{1 / q}<\infty\right\} .
$$

$L^{p, q}(\partial \Omega \times \mathbf{R})=L^{q}\left(\mathbf{R}, L^{p}(\partial \Omega)\right)$ is defined in a similar manner. In this section, we prove the following main results in this paper.

Theorem 1.1. Let $g \in L^{p, q}\left(\Sigma_{T}\right), 2 \leq p<\infty, 1<q<\infty$. Then there exists a unique solution $u$ on $\Omega_{T}$ satisfying (0.1), (0.2), and $\left\|(u)^{*}\right\|_{L^{p, q}\left(\Sigma_{T}\right)}<\infty$. Moreover, the solution $u$ can be represented in terms of a double layer potential and satisfies

$$
\left\|(u)^{*}\right\|_{L^{p, q}\left(\Sigma_{T}\right)} \leq C\|g\|_{L^{p, q}\left(\Sigma_{T}\right)} .
$$

Theorem 1.2. Let $g \in L^{p, q}\left(\Sigma_{T}\right), 1<p \leq 2,1<q<\infty$. Then there exists a unique solution $u$ on $\Omega_{T}$ satisfying $(0.1),(0.3)$, and $\left\|(\nabla u)^{*}\right\|_{L^{p, q}\left(\Sigma_{T}\right)}<\infty$. Moreover, $u$ can be represented in terms of a single-layer potential and satisfies

$$
\left\|(\nabla u)^{*}\right\|_{L^{p, q}\left(\Sigma_{T}\right)}+\left\|\left(\partial_{t}^{1 / 2} u\right)^{*}\right\|_{L^{p, q}\left(\Sigma_{T}\right)} \leq C\|g\|_{L^{p, q}\left(\Sigma_{T}\right)} .
$$

Definition 1.3. In Theorems 1.1 and 1.2 and throughout this paper, ( $)^{*}$ denotes the parabolic nontangential maximal function defined by

$$
\begin{aligned}
(u)^{*}(P, t)=\sup \{|u(X, s)|: & (X, s) \in \Omega_{T}(\text { or } \Omega \times \mathbf{R}) \\
& \left.|X-P|+|t-s|^{1 / 2}<2 \operatorname{dist}(X, \partial \Omega)\right\}
\end{aligned}
$$

for $(P, t) \in \partial \Omega \times(0, T)$ (or $\partial \Omega \times \mathbf{R}) . \quad \partial_{t}^{1 / 2} u$ denotes the half of a time derivative of $u$ defined by

$$
\partial_{t}^{1 / 2} u(X, t)=\frac{1}{\sqrt{\pi}} \partial_{t} \int_{-\infty}^{t} \frac{u(X, s)}{(t-s)^{1 / 2}} d s
$$


Remark 1.4. An example in [B1, Example 1.7, p. 344] shows that the ranges of $p, q$ in Theorems 1.1 and 1.2 are sharp, save possibly the end points $q=1$ and $\infty$. On the other hand, given a Lipschitz domain $\Omega$, there exists $e=e(\Omega)>0$, such that the initial Dirichlet problem is solvable for $2-e<p<\infty, 1<q<\infty$ and the initial Neumann problem is solvable for $1<p<2+e, 1<q<\infty$. This follows from the proof of Theorems 1.1 and 1.2 and a perturbation theorem of David and Semmes (unpublished, see [DKV] for a statement of their result). Also, Fabes has noted that the density of caloric measure in a Lipschitz cylinder lies in $L^{2}\left(\partial \Omega ; L^{\infty}(0, T)\right) \subset L^{2, \infty}\left(\Sigma_{T}\right)$. Hence, the initial Dirichlet problem is solvable in the dual space $L^{2,1}$. Fabes's observation is proven using the comparison principle for caloric functions (see [FGS]).

Let $f \in L^{p, q}\left(\Sigma_{T}\right), 1<p, q<\infty$, and let

$$
\mathscr{D}(f)(X, t)=\int_{0}^{t} \int_{\partial \Omega} \frac{\partial}{\partial N(Q)} \Gamma(X-Q, t-s) f(Q, s) d Q d s
$$

and

$$
\mathscr{S}(f)(X, t)=\int_{0}^{t} \int_{\partial \Omega} \Gamma(X-Q, t-s) f(Q, s) d Q d s
$$

be the double-layer potential and single-layer potential for the heat equation (0.1) respectively where $\Gamma(X, t)$ denotes the fundamental solution of the heat equation given by

$$
\Gamma(X, t)= \begin{cases}\frac{1}{(4 \pi t)^{n / 2}} e^{-|X|^{2} / 4 t}, & t>0, \\ 0, & t \leq 0 .\end{cases}
$$

Theorem 1.5. Let $1<p, q<\infty$. Then

$$
\begin{gathered}
\left\|(\mathscr{D}(f))^{*}\right\|_{L^{p, q}\left(\Sigma_{T}\right)}+\left\|(\nabla \mathscr{S}(f))^{*}\right\|_{L^{p, q}\left(\Sigma_{T}\right)}+\left\|\left(\partial_{t}^{1 / 2} \mathscr{S}(f)\right)^{*}\right\|_{L^{p, q}\left(\Sigma_{T}\right)} \leq C\|f\|_{L^{p, q}\left(\Sigma_{T}\right)}, \\
\frac{\partial}{\partial N} \mathscr{S}(f)_{ \pm}\left|\Sigma_{T}=\left( \pm \frac{1}{2} I+K\right) f, \quad \mathscr{D}(f)_{ \pm}\right|_{\Sigma_{T}}=\left(\mp \frac{1}{2} I+\widetilde{K}\right) f
\end{gathered}
$$

where \pm indicates the nontangential limits taken inside $\Omega_{T}$ and outside $\Omega \times \mathbf{R}$ respectively, $I$ denotes the identity operator, $K$ is a bounded singular integral operator on $L^{p, q}\left(\Sigma_{T}\right), \widetilde{K}=\Lambda K^{*} \Lambda$, and $\Lambda: L^{p, q}\left(\Sigma_{T}\right) \rightarrow L^{p, q}\left(\Sigma_{T}\right)$ is defined by $\Lambda(f)(P, t)=f(P, T-t)$.

Proof. The proof can be carried out using the theorem of Coifman, McIntosh, and Meyer on the Cauchy integral on Lipschitz curves [CMM], a variant of Fefferman-Stein's results on maximal functions [FSt], and the argument of Fabes-Riviere [FR]. The estimates are standard but lengthy. We omit the details here.

By Theorem 1.5, the existence of and estimates for solutions in Theorems 1.1 and 1.2 will follow if $\pm \frac{1}{2} I+K: L^{p, q}\left(\Sigma_{T}\right) \rightarrow L^{p, q}\left(\Sigma_{T}\right)$ is invertible for $1<p \leq 2,1<q<\infty$. To study the invertibility of $\pm \frac{1}{2} I+K$ on $L^{p, q}$, we shall use a vector-valued Calderón-Zygmund argument. To do this, we find it convenient to consider the equation

$$
\frac{\partial u}{\partial t}+u-\Delta u=0 \text { in } \Omega \times \mathbf{R} .
$$


It is easy to see that $e^{-t} \Gamma(X, t)$ is the fundamental solution for (1.6). Let

$$
\widetilde{\mathscr{S}}(f)(X, t)=\int_{-\infty}^{t} \int_{\partial \Omega} e^{-(t-s)} \Gamma(X-Q, t-s) f(Q, s) d Q d s
$$

be the single-layer potential for the equation (1.6). Then

$$
\left.\frac{\partial}{\partial N} \widetilde{\mathscr{S}}_{ \pm}(f)\right|_{\partial \Omega \times \mathbf{R}}=\left( \pm \frac{1}{2} f+e^{-t} K\left(e^{t} f\right)\right) .
$$

We shall first show that $\pm \frac{1}{2} I+e^{-t} K e^{t}$ is invertible on $L^{q}\left(\mathbf{R}, L^{p}(\partial \Omega)\right)$ for $1<p \leq 2,1<q<\infty$.

We begin with a uniqueness result. This result is proven in [B2, Theorems 5.2 and 5.4].

Lemma 1.7. Suppose that $u$ is a solution of (1.6) in $\Omega \times(-\infty, T)$ with $(u)^{*}+$ $(\nabla u)^{*} \in L^{q}\left(-\infty, T, L^{p}(\partial \Omega)\right)$ for some $T \in \mathbf{R}$ and $1<p, q<\infty$. Assume that either $\left.u\right|_{\partial \Omega \times(-\infty, T)}=0$ or $\left.(\partial u / \partial N)\right|_{\partial \Omega \times(-\infty, T)}=0$. Then $u \equiv 0$ in $\Omega \times(-\infty, T)$.

We also have uniqueness in $c \bar{\Omega} \times(-\infty, T)$, if, in addition, we assume that $|u(X, t)|=O\left(|X|^{2-n}\right)$ uniformly in $t$ as $|X| \rightarrow \infty$.

Theorem 1.8. $\pm \frac{1}{2} I+e^{-t} K e^{t}$ is invertible on $L^{p}(\partial \Omega \times \mathbf{R})$ for $1<p<2$.

Proof. The proof is essentially the same as that of [B2, Theorem 5.20, p. 39]. We only give a sketch here.

Taking the partial Fourier transform in the $t$ variable of both sides of equation (1.6), we obtain

$$
(1-i \tau) v-\Delta v=0 \text { in } \Omega, \tau \in \mathbf{R},
$$

where $v(X)=\hat{u}(X, \tau)=\int_{\mathbf{R}} e^{i t \tau} u(X, t) d t$. Let

$$
\Gamma_{\tau}(X)=\int_{0}^{\infty} e^{-(1+i \tau) t} \Gamma(X, t) d t
$$

denote the fundamental solution for (1.9) and

$$
v_{\tau}(X)=\int_{\partial \Omega} \Gamma_{\tau}(X-Q) h(Q) d Q \quad \text { for } h \in L^{p}(\partial \Omega), 1<p<\infty .
$$

Then

$$
\frac{\partial u_{\tau \pm}}{\partial N}=\left( \pm \frac{1}{2} I+K(\tau)\right)(h) \text { on } \partial \Omega \text {. }
$$

It follows from Rellich identities (see [B1, BS1, Proposition 2.2]) that

$$
\begin{aligned}
& c\left\{\left\|\nabla_{\tan } v_{\tau}\right\|_{L^{2}(\partial \Omega)}+\left\|(1+|\tau|)^{1 / 2} v_{\tau}\right\|_{L^{2}(\partial \Omega)}\right\} \\
& \quad \leq\left\|\frac{\partial v_{\tau}}{\partial N}\right\|_{L^{2}(\partial \Omega)} \leq C\left\{\left\|\nabla_{\tan } v_{\tau}\right\|_{L^{2}(\partial \Omega)}+\left\|(1+|\tau|)^{1 / 2} v_{\tau}\right\|_{L^{2}(\partial \Omega)}\right\} .
\end{aligned}
$$

This, together with a simple approximation argument, implies that

$$
\pm \frac{1}{2} I+K(\tau): L^{2}(\partial \Omega) \rightarrow L^{2}(\partial \Omega)
$$

is invertible and

$$
\left\|\left( \pm \frac{1}{2} I+K(\tau)\right)^{-1}\right\|_{L^{2}(\partial \Omega)-L^{2}(\partial \Omega)} \leq C
$$


where $C$ is independent of $\tau \in \mathbf{R}$. Note that

$$
\left\{\left( \pm \frac{1}{2} I+e^{-t} K e^{t}\right)(f)(P, \cdot)\right\}^{\wedge}(\tau)=\left( \pm \frac{1}{2} I+K(\tau)\right)\left(f^{\wedge}(\cdot, \tau)\right)(P) .
$$

The invertibility of $\pm \frac{1}{2} I+e^{-t} K e^{t}$ on $L^{2}(\partial \Omega \times \mathbf{R})$ then follows easily from (1.10) and Plancherel's theorem.

Let $\Omega_{+}=\Omega$ and $\Omega_{-}={ }^{c} \bar{\Omega}$. Let $L_{1}^{p}(\partial \Omega \times \mathbf{R})$ denote the closure of the space

$$
\left\{v: v=\left.u\right|_{\partial \Omega \times \mathbf{R}}, u \in C_{c}^{\infty}\left(\mathbf{R}^{n} \times \mathbf{R}\right)\right\}
$$

with respect to the norm

$$
\|v\|_{L_{1}^{p}(\partial \Omega \times \mathbf{R})}=\left\|\nabla_{\tan } v\right\|_{L^{p}(\partial \Omega \times \mathbf{R})}+\left\|\partial_{t}^{1 / 2} v\right\|_{L^{p}(\partial \Omega \times \mathbf{R})}+\|v\|_{L^{p}(\partial \boldsymbol{\Omega} \times \mathbf{R})} .
$$

As in [B2], to establish the invertibility of $\pm \frac{1}{2} I+e^{-t} K e^{t}$ on $L^{p}(\partial \Omega \times \mathbf{R})$ for $1<p<2$, we need to consider the Neumann problem on $\Omega_{ \pm} \times \mathbf{R}$ with $L^{p}$ data:

$$
\begin{cases}\frac{\partial u}{\partial t}+u-\Delta u=0 & \text { in } \Omega_{ \pm} \times \mathbf{R}, \\ \frac{\partial u}{\partial N}=g \in L^{p}(\partial \Omega \times \mathbf{R}) & \text { on } \mathbf{R}, \\ (u)^{*}+(\nabla u)^{*} \in L^{p}(\partial \Omega \times \mathbf{R}), & \end{cases}
$$

and the Dirichlet problem on $\Omega_{ \pm} \times \mathbf{R}$ with $L_{1}^{p}$ data:

$$
\begin{cases}\frac{\partial u}{\partial t}+u-\Delta u=0 & \text { in } \Omega_{ \pm} \times \mathbf{R}, \\ u=g \in L_{1}^{p}(\partial \Omega \times \mathbf{R}) & \text { on } \partial \Omega \times \mathbf{R}, \\ (u)^{*}+(\nabla u)^{*} \in L^{p}(\partial \Omega \times \mathbf{R}) . & \end{cases}
$$

It can be shown that, given $g \in L^{p}(\partial \Omega \times \mathbf{R}), 1<p \leq 2$, there exists a unique solution $u$ satisfying (1.1) and we have

$$
\left\|(\nabla u)^{*}\right\|_{L^{p}(\partial \mathbf{\Omega} \times \mathbf{R})}+\|u\|_{L_{1}^{p}(\partial \Omega \times \mathbf{R})} \leq C\|g\|_{L^{p}(\partial \Omega \times \mathbf{R})} .
$$

Also, given $g \in L_{1}^{p}(\partial \Omega \times \mathbf{R}), 1<p \leq 2$, there exists a unique solution $u$ satisfying (1.12). Moreover, the solution to (1.12) satisfies the estimates

$$
\left\|(\nabla u)^{*}\right\|_{L^{p}(\partial \Omega \times \mathbf{R})} \leq C\|g\|_{L_{1}^{p}(\partial \Omega \times \mathbf{R})} .
$$

The above results follow by interpolation from the $L^{2}$-case and estimates of solutions with atomic data. The estimates of solutions with atomic data can be established using the $L^{2}$-estimates and estimates on Green's functions for (1.6) in $\Omega_{ \pm} \times \mathbf{R}$.

In fact, let $G(X, Y, t-s)$ be the Green's functions for the heat equation $(0.1)$ in $\Omega \times \mathbf{R}$ with Neumann boundary condition. Clearly, $G_{1}(X, Y, t-s)=$ $e^{-(t-s)} G(X, Y, t-s)$ is the Green's function for the equation (1.6) in $\Omega \times \mathbf{R}$. By well-known estimates on $G(X, Y, t)$, we have

$$
\begin{gathered}
\left|G_{1}(X, Y, t)\right| \leq C e^{-t} \text { if } t \geq 1, \\
\left|G_{1}(X, Y, t)\right| \leq \frac{c}{t^{n / 2}} e^{-|X-Y|^{2} / c t}, \quad t>0, \\
\left|G_{1}\left(X, Y_{1}, t-s_{1}\right)-G_{1}\left(X, Y_{2}, t-s_{2}\right)\right| \leq \frac{c\left(\left|Y_{1}-Y_{2}\right|+\left|t_{1}-t_{2}\right|^{1 / 2}\right)^{\delta_{0}}}{\left(\left|X-Y_{1}\right|+\left|t-s_{1}\right|^{1 / 2}\right)^{n+\delta_{0}}}
\end{gathered}
$$


for some $\delta_{0}=\delta_{0}(\Omega)>0$, if

$$
\left|Y_{1}-Y_{2}\right|+\left|s_{1}-s_{2}\right|^{1 / 2}<\frac{1}{10}\left[\left|X-Y_{1}\right|+\left|t-s_{1}\right|^{1 / 2}\right]
$$

Now, let $u$ be a solution to (1.11) with atomic data, i.e.,

$$
\frac{\partial u}{\partial N}=a, \quad \operatorname{supp} a \subset \Delta\left(P_{0}, r\right) \times\left(t_{0}-r^{2}, t_{0}\right)
$$

for some $\left(P_{0}, t_{0}\right) \in \partial \Omega \times \mathbf{R}$ and $r>0, \iint_{\partial \Omega \times \mathbf{R}} a=0$, and $\|a\|_{L^{2}(\partial \Omega \times \mathbf{R})} \leq$ $r^{(n+1) / 2}$ where $\Delta\left(P_{0}, r\right)=\left\{Q \in \partial \Omega,\left|Q-P_{0}\right|<r\right\}$. Then

$$
u(X, t)=\int_{-\infty}^{t} \int_{\partial \Omega} G(X, Q, t-s) a(Q, s) d Q d s
$$

and, if $t>t_{0}$,

$$
u(X, t)=\int_{t_{0}-r^{2}}^{t_{0}} \int_{\Delta\left(P_{0}, r\right)}\left[G(X, Q, t-s)-G\left(X, P_{0}, t-t_{0}\right)\right] a(Q, s) d Q d s
$$

It follows from (1.15)-(1.17) that

$$
|u(X, t)| \leq \begin{cases}0, & t \leq t_{0}-r^{2}, \\ \frac{c \delta_{0}}{\left(\left|X-P_{0}\right|+\left|t-t_{0}\right|^{1 / 2}\right)^{n+\delta_{0}}}, & t_{0}-r^{2}<t \leq t_{0}+10 \\ c e^{-t}, & t>t_{0}+10 .\end{cases}
$$

The required estimates on solutions with atomic data follow from (1.18) and $L^{2}$-estimates in the same fashion as in [B2, Lemma 3.1, p. 16].

Finally, let $f \in L^{p}(\partial \Omega \times \mathbf{R}), 1<p<2$, and $u=\mathscr{S}(f)$. Then

$$
f=\frac{\partial u^{+}}{\partial N}-\frac{\partial u^{-}}{\partial N}
$$

Thus, by the solvability of (1.11), (1.12) and estimates (1.13), (1.14),

$$
\begin{aligned}
\|f\|_{L^{p}(\partial \Omega \times \mathbf{R})} & \leq\left\|\frac{\partial u^{+}}{\partial N}\right\|_{L^{p}(\partial \Omega \times \mathbf{R})}+\left\|\frac{\partial u^{-}}{\partial N}\right\|_{L^{p}(\partial \Omega \times \mathbf{R})} \\
& \leq\left\|\frac{\partial u^{+}}{\partial N}\right\|_{L^{p}(\partial \Omega \times \mathbf{R})}+C\|u\|_{L_{1}^{p}(\partial \Omega \times \mathbf{R})} \\
& \leq C\left\|\frac{\partial u^{+}}{\partial N}\right\|_{L^{p}(\partial \Omega \times \mathbf{R})}=C\left\|\left(\frac{1}{2} I+e^{-t} K e^{t}\right) f\right\|_{L^{p}(\partial \Omega \times \mathbf{R})} .
\end{aligned}
$$

Hence, to show $\frac{1}{2} I+e^{-t} K e^{t}: L^{p}(\partial \Omega \times \mathbf{R}) \rightarrow L^{p}(\partial \Omega \times \mathbf{R})$ is invertible, it suffices to prove that the range of $\frac{1}{2} I+e^{-t} K e^{t}$ is dense in $L^{p}(\partial \Omega \times \mathbf{R})$. To this end, let $g \in C_{0}^{\infty}\left(\mathbf{R}^{n} \times \mathbf{R}\right)$. Since $\frac{1}{2} I+e^{-t} K e^{t}$ is invertible on $L^{2}(\partial \Omega \times \mathbf{R})$, there exists $f \in L^{2}(\partial \Omega \times \mathbf{R})$ such that

$$
\left(\frac{1}{2} I+e^{-t} K e^{t}\right) f=g .
$$

Let $u=\widetilde{\mathscr{S}}(f)$ and $v$ be a solution of (1.11) in $\Omega \times \mathbf{R}$ such that $\partial v / \partial N=g$ and $(v)^{*}+(\nabla v)^{*} \in L^{p}(\partial \Omega \times \mathbf{R})$. Since $u \equiv 0$ on $\Omega \times\left(-\infty, T_{0}\right)$ for some $T_{0} \in \mathbf{R}$ by Lemma 1.7 , we have

$$
(\nabla(u-v))^{*}+(u-v)^{*} \in L^{p}(\partial \Omega \times(-\infty, T))
$$


for any $T \in \mathbf{R}$. It again follows from Lemma 1.7 that $u \equiv v$ on $\Omega \times \mathbf{R}$. Hence,

$$
\left\|\frac{\partial u^{+}}{\partial N}\right\|_{L^{p}(\partial \Omega \times \mathbf{R})}<\infty \text {. }
$$

Repeating the above argument in $c \overline{\mathbf{\Omega}} \times \mathbf{R}$, we get $\left\|\partial u^{-} / \partial N\right\|_{L^{p}(\partial \boldsymbol{\Omega} \times \mathbf{R})}<\infty$. Thus,

$$
\|f\|_{L^{p}(\partial \boldsymbol{\Omega} \times \mathbf{R})} \leq\left\|\frac{\partial u^{+}}{\partial N}\right\|_{L^{p}(\partial \boldsymbol{\Omega} \times \mathbf{R})}+\left\|\frac{\partial u^{-}}{\partial N}\right\|_{L^{p}(\partial \boldsymbol{\Omega} \times \mathbf{R})}<\infty,
$$

i.e., $f \in L^{p}(\partial \Omega \times \mathbf{R})$. Hence, $\frac{1}{2} I+e^{-t} K e^{t}$ is invertible on $L^{p}(\partial \Omega \times \mathbf{R})$. The proof of the invertibility of $-\frac{1}{2} I+e^{-t} K e^{t}$ is similar.

We now study the invertibility of $\pm \frac{1}{2} I+e^{-t} K e^{t}$ on mixed norm spaces.

Theorem 1.19. Let $1<p \leq 2,1<q<\infty$. Then $\pm \frac{1}{2} I+e^{-t} K e^{t}: L^{p, q}(\Omega \times \mathbf{R}) \rightarrow$ $L^{p, q}(\partial \Omega \times \mathbf{R})$ is invertible.

Proof. We give the proof for $\frac{1}{2} I+e^{-t} K e^{t}$. The invertibility of $-\frac{1}{2} I+e^{-t} K e^{t}$ follows in the same manner.

Let $S=\frac{1}{2} I+e^{-t} K e^{t}$. Recall that

$$
[S(f)(P, \cdot)]^{\wedge}(\tau)=\left(\frac{1}{2} I+K(\tau)\right)\left(f^{\wedge}(\cdot, \tau)\right)(P)
$$

for $\tau \in \mathbf{R}$ and $f \in L^{2}(\partial \Omega \times \mathbf{R})$. Let $m(\tau)=\frac{1}{2} I+K(\tau), \tau \in \mathbf{R}$. It follows from the theorem of Coifman, McIntosh, and Meyer [CMM] that

$$
\|m(\tau)\|_{L^{p}(\partial \Omega) \rightarrow L^{p}(\partial \Omega)} \leq C \text { for } 1<p<\infty
$$

where $C$ is a constant independent of $\tau$. Moreover, it is not difficult to show that

$$
\left|\frac{d^{\alpha}}{d \tau^{\alpha}} m(\tau) h(P)\right| \leq \frac{C_{\alpha}}{(1+|\tau|)^{\alpha}} M_{\partial \Omega}(h)(P)
$$

for any integer $\alpha \geq 1$, where $h \in L^{p}(\partial \Omega)$ and $M_{\partial \Omega}$ denotes the HardyLittlewood maximal function on $\partial \Omega$. Thus

$$
\left\|\frac{d^{\alpha}}{d \tau^{\alpha}} m(\tau)\right\|_{L^{p(\partial \Omega) \rightarrow L^{p}(\partial \Omega)}} \leq \frac{C_{\alpha}}{(1+|\tau|)^{\alpha}}
$$

for any integer $\alpha \geq 0$. From (1.10), we know

$$
\left\|m^{-1}(\tau)\right\|_{L^{2}(\partial \Omega) \rightarrow L^{2}(\partial \Omega)} \leq C .
$$

We claim that

$$
\left\|m^{-1}(\tau)\right\|_{L^{p}(\partial \Omega) \rightarrow L^{p}(\partial \Omega)} \leq C \text { for } 1<p \leq 2 .
$$

To prove the claim (1.21), let $h_{1}, h_{2} \in C_{c}^{\infty}\left(\mathbf{R}^{n}\right)$. By (1.23) and (1.22), $\left\langle m^{-1}(\tau) h_{1}, h_{2}\right\rangle$ is a bounded continuous function of $\tau$, where $\langle$,$\rangle denotes$ the inner product in $L^{2}(\partial \Omega)$. Hence,

$$
\begin{aligned}
\left\langle m^{-1}(\tau) h_{1}, h_{2}\right\rangle & =\frac{1}{\pi} \lim _{\varepsilon \rightarrow 0^{+}} \frac{1}{\sqrt{\varepsilon}} \int_{\mathbf{R}} e^{-(\sigma-r)^{2} / 2 \varepsilon}\left\langle m^{-1}(\tau) h_{1}, h_{2}\right\rangle d \sigma \\
& =\frac{1}{\pi} \lim _{\varepsilon \rightarrow 0^{+}} \frac{1}{\sqrt{\varepsilon}} \int_{\mathbf{R}}\left\langle m^{-1}(\sigma) e^{-(\sigma-\tau)^{2} / 2 \varepsilon} h_{1}, e^{-(\sigma-\tau)^{2} / 2 \varepsilon} h_{2}\right\rangle d \sigma \\
& =\frac{1}{\pi} \lim _{\varepsilon \rightarrow 0} \frac{1}{\sqrt{\varepsilon}} \int_{\mathbf{R}}\left\langle S^{-1}\left(f_{1}\right), f_{2}\right\rangle d t
\end{aligned}
$$


where $f_{1}=(\sqrt{\varepsilon} / \sqrt{2 \pi}) e^{-\varepsilon t^{2} / 2} \cdot e^{i t \tau} h_{1}$ and $f_{2}=(\sqrt{\varepsilon} / \sqrt{2 \pi}) e^{-\varepsilon t^{2} / 2} \cdot e^{i t \tau} h_{2}$. Since $S^{-1}: L^{p}\left(\mathbf{R}, L^{p}(\partial \Omega)\right) \rightarrow L^{p}\left(\mathbf{R}, L^{p}(\partial \Omega)\right.$ ) is bounded for $1<p \leq 2$ (Theorem $1.8)$, we have

$$
\begin{aligned}
\left\|\int_{\mathbf{R}}\left\langle S^{-1}\left(f_{1}\right), f_{2}\right\rangle d t\right\| & \leq \int_{\mathbf{R}}\left\|S^{-1}\left(f_{1}\right)\right\|_{L^{p}(\partial \Omega)}\left\|f_{2}\right\|_{L^{p^{\prime}}(\partial \Omega)} d t \\
& \leq\left\|S^{-1}\left(f_{1}\right)\right\|_{L^{p}\left(\mathbf{R}, L^{p}(\partial \Omega)\right)}\left\|f_{2}\right\|_{L^{p^{\prime}}\left(\mathbf{R}, L^{p^{\prime}}(\partial \Omega)\right)} \\
& \leq C\left\|f_{1}\right\|_{L^{p}\left(\mathbf{R}, L^{p}(\partial \Omega)\right)}\left\|f_{2}\right\|_{L^{p^{\prime}}\left(\mathbf{R}, L^{p^{\prime}}(\partial \boldsymbol{\Omega})\right)} \\
& \leq C \sqrt{\varepsilon}\left\|h_{1}\right\|_{L^{p}(\partial \Omega)}\left\|h_{2}\right\|_{L^{p^{\prime}}(\partial \boldsymbol{\Omega})} .
\end{aligned}
$$

Thus, we have proved that

$$
\left|\left\langle m^{-1}(\tau) h_{1}, h_{2}\right\rangle\right| \leq C\left\|h_{1}\right\|_{L^{p}(\partial \Omega)}\left\|h_{2}\right\|_{L^{p^{\prime}}(\partial \Omega)} .
$$

Claim (1.24) then follows by duality.

Now, fix $p \in(1,2)$. Let $\mathbf{B}=L^{p}(\partial \Omega)$. By (1.24) and (1.21),

$$
\left\|\frac{d^{\alpha}}{d \tau^{\alpha}} m^{-1}(\tau)\right\|_{\mathbf{B} \rightarrow \mathbf{B}} \leq \frac{C_{\alpha}}{(1+|\tau|)^{\alpha}}
$$

for any integer $\alpha \geq 0$. Since $\left[S^{-1}(f)\right]^{\wedge}(\tau)=m^{-1}(\tau) f^{\wedge}(\tau)$, it follows from a standard Calderón-Zygmund argument that $S^{-1}$, as an operator on functions with values in $\mathbf{B}$, is associated with an $\mathscr{L}(\mathbf{B})$-valued Calderón-Zygmund kernel, where $\mathscr{L}(\mathbf{B})$ denotes the spaces of bounded linear operators on $\mathbf{B}$. But $S^{-1}: L^{p}(\mathbf{R}, \mathbf{B}) \rightarrow L^{p}(\mathbf{R}, \mathbf{B})$ is bounded, so by the standard Calderón-Zygmund theory,

$$
S^{-1}: L^{q}(\mathbf{R}, \mathbf{B}) \rightarrow L^{q}(\mathbf{R}, \mathbf{B})
$$

is bounded for $1<q<\infty$.

Corollary 1.25. Let $0<T<\infty$. Then $\pm \frac{1}{2} I+K: L^{p, q}\left(\Sigma_{T}\right) \rightarrow L^{p, q}\left(\Sigma_{T}\right)$ is invertible for $1<p \leq 2,1<q<\infty$.

Proof. We give the proof for $\frac{1}{2} I+K$. The invertibility of $-\frac{1}{2} I+K$ follows in the same manner.

Given $g \in L^{p, q}\left(\Sigma_{T}\right), 1<p \leq 2,1<q<\infty$. Let $\tilde{g}$ be the extension of $g$ by zero to $\partial \Omega \times \mathbf{R}$. Clearly, $e^{-t} \tilde{g} \in L^{p, q}(\partial \Omega \times \mathbf{R})$. Hence, by Theorem 1.19, there exists $F \in L^{p, q}(\partial \Omega \times \mathbf{R})$ such that $\left(\frac{1}{2} I+e^{-t} K e^{t}\right)(F)=e^{-t} \tilde{g}$ on $\partial \Omega \times \mathbf{R}$ and

$$
\|F\|_{L^{p, q}(\partial \Omega \times \mathbf{R})} \leq C\left\|e^{-t} \tilde{g}\right\|_{L^{p, q}(\partial \Omega \times \mathbf{R})} \leq C\|g\|_{L^{p, q}\left(\Sigma_{T}\right)} .
$$

Since $e^{-t} \tilde{g}(P, t)=0$ for $t \leq 0$, it follows from Lemma 1.7 that $F(P, t)=0$ on $\partial \Omega \times(-\infty, 0)$. Now, let $f=\left.e^{t} F\right|_{\Sigma_{T}}$. Then $\left(\frac{1}{2} I+K\right) f=g$ on $\Sigma_{T}$. Moreover,

$$
\|f\|_{L^{p, q}\left(\Sigma_{T}\right)} \leq C_{T}\|F\|_{L^{p, q\left(\Sigma_{T}\right)}} \leq C_{T}\|g\|_{L^{p, q\left(\Sigma_{T}\right)}}
$$

where $C_{T}$ depends on $p, q, \partial \Omega, n$, and $T$.

Finally, we are ready to prove our main results-Theorems 1.1 and 1.2.

Proof of Theorem 1.1. Let $f \in L^{p, q}\left(\Sigma_{T}\right)$ and $u=\mathscr{D}(f)$. Then $\left.u\right|_{\Sigma_{T}}=$ $\Lambda\left(-\frac{1}{2} I+K\right)^{*} \Lambda(f)$ (Theorem 1.5). By Corollary 1.25, $\Lambda\left(-\frac{1}{2} I+K\right)^{*} \Lambda$ : $L^{p, q}\left(\Sigma_{T}\right) \rightarrow L^{p, q}\left(\Sigma_{T}\right)$ is invertible for $2 \leq p<\infty, 1<q<\infty$. The existence then follows. 
To show the uniqueness, we construct a Green's function

$$
G(X, Y, t)=\Gamma(X-Y, t)-V(X, Y, t)
$$

for $X \in \bar{\Omega}, Y \in \Omega$, where

$$
\begin{aligned}
V(X, Y, t)=\int_{0}^{t} \int_{\partial \Omega} \Gamma(X-Q, t-s)\left(-\frac{1}{2} I+K\right)^{-1} \\
\times\left(\frac{\partial}{\partial N} \Gamma(Y-\cdot, \cdot)\right)(Q, s) d Q d s .
\end{aligned}
$$

Then the argument of Fabes and Riviere in [FR, Theorem 2.3, p. 188] may go through with obvious modifications. We omit the details.

Proof of Theorem 1.2. The existence follows from the invertibility of $\frac{1}{2} I+K$ on $L^{p, q}\left(\Sigma_{T}\right)$ for $1<p \leq 2$ and $1<q<\infty$, while the uniqueness is contained in Lemma 1.7.

\section{REFERENCES}

[B1] R. M. Brown, The method of layer potentials for the heat equation in Lipschitz cylinders, Amer. J. Math. 111 (1989), 330-379.

[B2] - The initial-Neumann problem for the heat equation in Lipschitz cylinders, Trans. Amer. Math. Soc. 320 (1990), 1-52.

[BS1] R. M. Brown and Z. Shen, The initial-Dirichlet problem for a fourth-order parabolic equation in Lipschitz cylinders, Indiana Univ. J. Math. 39 (1990), 1313-1353.

[BS2] _ Boundary value problems in Lipschitz cylinders for three-dimensional parabolic systems, Revista Mat. Iberó. 8 (1992), 271-303.

[CMM] R. R. Coifman, A. McIntosh, and Y. Meyer, L'intégrale de Cauchy définit un opérateur borné sur $L^{2}$ pour les courbes Lipschitziennes, Ann. of Math. (2) 116 (1982), 361-387.

[DK] B. E. J. Dahlberg and C. E. Kenig, Hardy spaces and the Neumann problem in $L^{p}$ for Laplace's equation in Lipschitz domains, Ann. of Math. (2) 125 (1987), 437-465.

[FR] E. B. Fabes and N. M. Rivière, Dirichlet and Neumann problems for the heat equation in $C^{1}$-cylinders, Proc. Sympos. Pure Math., vol. 35, Amer. Math. Soc., Providence, RI, 1979, pp. 179-196.

[FGS] E. B. Fabes, N. Garofalo, and S. Salsa, A backward Harnack inequality and Fatou theorem for nonnegative solutions of parabolic equations, Illinois J. Math. 30 (1986), 536-565.

[FS] E. B. Fabes and S. Salsa, Estimates of caloric measure and the initial-Dirichlet problem for the heat equation in Lipschitz cylinders, Trans. Amer. Math. Soc. 279 (1983), 635-650.

[FSt] C. Fefferman and E. M. Stein, Some maximal inequalities, Amer. J. Math. 93 (1971), 107115.

[JK] D. Jerison and C. E. Kenig, The Neumann problem on Lipschitz domains, Bull. Amer. Math. Soc. (N.S.) 4 (1981), 703-707.

[K] C. E. Kenig, Elliptic boundary value problems on Lipschitz domains, Ann. of Math. Stud., vol. 112, Princeton Univ. Press, Princeton, NJ, 1986, pp. 131-183.

[NSt] A. Nagel and E. M. Stein, Lectures on pseudo-differential operators, Princeton Univ. Press, Princeton, NJ, 1979.

[S] Z. Shen, Boundary value problems for parabolic Lamé systems and a nonstationary linearized system of Navier-Stokes equations in Lipschitz cylinders, Amer. J. Math. 113 (1991), 293373. 
[St] E. M. Stein, Singular integrals and differentiability properties of functions, Princeton Univ. Press, Princeton, NJ, 1970.

[V] G. Verchota, Layer potentials and regularity for the Dirichlet problem for Laplace's equation in Lipschitz domains, J. Funct. Anal. 59 (1984), 572-611.

DePartment of Mathematics, University of Kentucky, LeXington, Kentucky 40506

E-mail address: rbrown@ms.uky.edu

Department of Mathematics, Purdue University, West Lafayette, Indiana 47906

E-mail address: shenz@math.purdue.edu 